\title{
MULTI-FIDELITY STOCHASTIC COLLOCATION METHOD FOR COMPUTATION OF STATISTICAL MOMENTS*
}

\author{
XUEYU ZHU ${ }^{\dagger}$, ERIN M. LINEBARGER ${ }^{\ddagger}$, AND DONGBIN XIU $\$$
}

\begin{abstract}
We present an efficient numerical algorithm to approximate the statistical moments of stochastic problems, in the presence of models with different fidelities. The method extends the multi-fidelity approximation method developed in $[18,26]$. By combining the efficiency of low-fidelity models and the accuracy of high-fidelity models, our method exhibits fast convergence with a limited number of high-fidelity simulations. We establish an error bound of the method and present several numerical examples to demonstrate the efficiency and applicability of the multi-fidelity algorithm.
\end{abstract}

Key words. Uncertainty quantification, stochastic collocation, multi-fidelity

1. Introduction. In recent years, many efforts have been devoted to the development of efficient numerical methods for uncertainty quantification (UQ). In practical computations, the most widely used method is stochastic collocation, as it is nonintrusive sampling based and allows one to use existing deterministic codes. Unfortunately, this number of deterministic simulations required by accurate stochastic collocation methods grows very rapidly for high dimensional random inputs - the curse of dimensionality. For large scale simulations, the computational cost can become prohibitive, as the computation of each individual deterministic sample is highly costly. Many options have been investigated to tackle this challenge. For example, methods that explore more efficient sampling strategies using sparse grids, adaptivity, smoothness or sparsity of the solutions, cf. [25, 2, 1, 11, 5, 8, 9, 12, 16, 17, 19, 20, 23]. There is also a recent surge of interest in multilevel Monte Carlo method, which uses the hierarchy models by physical space refinement to achieve variance reduction in random space, cf., [14, 4, 3, 7, 21]. Other approaches to achieve variance reduction have also been presented, cf., $[6,22]$.

In this paper we focus primarily on the computation of solution statistics using models with different fidelities. In particular, we focus on the case with one highfidelity model and one low-fidelity model. Here, the high-fidelity model is able to produce high resolution solution to the underlying physical problem. The simulation cost is high, thus preventing us from using the standard sampling strategy (Monte Carlo, sparse grids, etc.). The low-fidelity model, on the other hand, is not highly accurate but can capture the essential behavior of the underlying problem. It is computationally cheap and can be sampled a large number of times. Typically, the low-fidelity models are constructed using simplified physics and/or much coarser discretization. Examples are abundant in many problems, for example, the fine-scale versus the coarse grained models in multi-scale problems.

We present an efficient stochastic collocation algorithm for computing solution statistics using a high-fidelity model and a low-fidelity model. A distinct feature of our method is that it "seperate" the low-fidelity solutions and high-fidelity solutions. It

${ }^{*}$ This work is partially supported by AFOSR FA95501410022, DARPA N660011524053, and NSF DMS 1418771.

${ }^{\dagger}$ Scientific Computing and Imaging Institute, University of Utah, Salt Lake City, UT 84112. Email: xzhu@sci.utah.edu.

¥ Department of Mathematics, University of Utah, Salt Lake City, UT 84112, USA. Email: aerinline@sci.utah.edu

${ }^{\S}$ Corresponding author. Department of Mathematics, The Ohio State University, Columbus, OH 43210, USA. Email: xiu.16@osu.edu. 
uses the low-fidelity solutions, which consist of a large number of samples, to construct a best approximation of the target solution statistics (mean, variance, etc), and then apply the best approximation to the high-fidelity samples. Our method is essentially a "learning" algorithm, where the low-fidelity samples are used to "train" the best approximation. It is different from most of the existing methods, which usually achieve improved performance via variance reduction or by exploring the hierarchical structure (if available) of the models. The current method is an extension of the method developed in $[18,26]$, where the same training idea was first proposed and used to predict the solutions at arbitrary sample locations. A straightforward way to use the technique of $[18,26]$ to compute solution statistics is to compute the bi-fidelity solutions at every sample points and then compute the statistics. Although computing each bi-fidelity solution is efficient, computing such solutions at a large number of samples becomes expensive. The major contribution of this paper is to present a mathematically equivalent algorithm that directly computes the solution statistics and bypasses the step of a large number of bi-fidelity computations. Here we show that the method can be highly efficient in approximating the statistics (mean, variance, etc) of the underlying stochastic problem. We establish an error bound of the method and use extensive numerical examples to demonstrate its performance. In the examples with varying multiple dimensions, accuracy solutions can be obtained by $O(10)$ number of high-fidelity simulation samples.

2. Problem Setup. Let $w$ be the solution of a system of governing equations in a bounded spatial domain $D \subset \mathbb{R}^{\ell}, \ell=1,2,3$, and a random parameter domain $I_{Z} \subseteq \mathbb{R}^{d}, d \geq 1$. For general discussion we do not assume any specific form of the governing equations. We are interested in a quantity-of-interest (QoI), which is a function of the solution $w$, i.e.,

$$
v=q(w): \bar{D} \times I_{Z} \rightarrow \mathbb{R}
$$

Hereafter we denote $x=\left(x_{1}, \ldots, x_{\ell}\right)$ the spatial variable and $z=\left(z_{1}, \ldots, z_{d}\right)$ the random variable. Let $\rho: I_{z} \rightarrow \mathbb{R}^{+}$be the probability distribution function of $z$. We are interested in evaluating the statistical average of the QoI, $\nu: \bar{D} \rightarrow \mathbb{R}$,

$$
\nu(\cdot)=\mathbb{E}[v]=\int v(\cdot, z) \rho(z) d z .
$$

For example, when $v=w^{k}, k \geq 1$, it stands for the $k$-th moment of the solution.

2.1. Numerical approximations. For numerical approximation, we seek an approximate solution $u$ in a linear subspace $V$ for any fixed random variables,

$$
u: I_{z} \rightarrow V \text {. }
$$

Obviously, the choice of the linear subspace $V$ depends on the chosen numerical method. We assume that the numerical method is deterministic and satisfies

$$
u(\cdot, z) \approx v(\cdot, z), \quad \forall z \in I_{Z},
$$

in a proper norm in the physical space.

Since the solution dependence in the random space can also be complex, the mean operator $\mathbb{E}$ in (2.2) also needs to be approximated. In this paper we focus on linear sampling based approximation, which is the predominant approach in practice. 
Let $\Theta=\left\{z_{1}, \ldots, z_{m}\right\} \subset I_{Z}$ be a set of samples, then for any integrable function $f: I_{Z} \rightarrow \mathbb{R}$ we define

$$
\widetilde{\mathbb{E}}[f ; \Theta]:=\sum_{i=1}^{m} w_{i} f\left(z_{i}\right) \approx \mathbb{E}[f]
$$

where $w_{i}$ is the weight at the sample $z_{i}$, for $i=1, \ldots, m$. For example, the standard Monte Carlo method has an uniform weight $w_{i} \equiv 1 / m$, whereas for most cubature rules the weights are non-uniform. Hereafter we assume the weights satisfy

$$
\sum_{i=1}^{m} w_{i}=1, \quad\|w\|_{\ell_{2}}<\infty
$$

where $\|w\|_{\ell_{2}}$ is the 2 -norm of $w=\left(w_{1}, \ldots, w_{m}\right)$. Although it is highly desirable to have $w_{i}>0$, this is not the case for many cubature rules.

With the approximations in both the physical space and the random space, we have

$$
\mu(\cdot)=\widetilde{\mathbb{E}}[u ; \Theta] \approx \nu(\cdot)
$$

as an approximation to the true statistical average (2.2).

2.2. High and low fidelity approximations. We assume there is a highfidelity deterministic numerical approximation

$$
u^{H}: I_{Z} \rightarrow V^{H}
$$

in a high-fidelity Hilbert subspace $V^{H}$, equipped with inner product $\langle\cdot, \cdot\rangle^{H}$ and its induced norm $\|\cdot\|^{H}$. Similarly, we assume there is a low-fidelity approximation

$$
u^{L}: I_{Z} \rightarrow V^{L},
$$

equipped with inner product $\langle\cdot, \cdot\rangle^{L}$ and norm $\|\cdot\|^{L}$. We assume that $u^{H}$ is much more accurate than $u^{L}$, but also much more time consuming to obtain.

Let $\Gamma_{N}=\left\{z_{1}, \cdots, z_{N}\right\} \subset I_{Z}$ be a set of dense samples, from which an accurate approximation of the expectation $\widetilde{\mathbb{E}}(2.4)$ can be obtained. We assume $N \gg 1$. We then readily have the low-fidelity estimation of the mean

$$
\mu^{L}(\cdot)=\widetilde{\mathbb{E}}\left[u^{L} ; \Gamma_{N}\right]=\sum_{i=1}^{N} w_{i} u^{L}\left(\cdot, z_{i}\right),
$$

which is not highly accurate due to the limited accuracy of $u^{L}$ at each sample points. The high-fidelity approximation $\widetilde{\mathbb{E}}\left[u^{H}\right]$

$$
\mu^{H}(\cdot)=\widetilde{\mathbb{E}}\left[u^{H} ; \Gamma_{N}\right]=\sum_{i=1}^{N} w_{i} u^{H}\left(\cdot, z_{i}\right)
$$

should be accurate, but it is not computable due to the exceedingly large simulation cost of $N \gg 1$. Our goal is to construct a bi-fidelity approximation $\mu^{B} \approx \mu^{H}$, by using the low-fidelity mean (2.9) and a limited number of high-fidelity samples. 
We remark that the method developed in $[18,26]$ allows one to compute bi-fidelity solutions at any given sample point, i.e., $u^{B}(z) \approx u^{H}(z), z \in I_{Z}$. It is then natural to approximate the high-fidelity mean by using the bi-fidelity solutions, i.e.,

$$
\widetilde{\mu}^{B}(\cdot)=\widetilde{\mathbb{E}}\left[u^{B} ; \Gamma_{N}\right]=\sum_{i=1}^{N} w_{i} u^{B}\left(\cdot, z_{i}\right)
$$

This straightforward approach requires the bi-fidelity approximations of the solution at the dense sample set $\Gamma_{N}$ and can become computationally expensive. In the following, we shall present a mathematically equivalent method that directly constructs the bi-fidelity mean and avoids the bi-fidelity approximation at the dense set $\Gamma_{N}$. The new method is thus much more efficient.

3. Bi-fidelity method for expectation. In this section we describe the bifidelity algorithm for computing the expectation (2.4). The method is an extension of the work of $[18,26]$, which developed a multi-fidelity approximation of the solution at any sample location $z$.

3.1. The main algorithm. Again, let $\Gamma_{N}=\left\{z_{1}, \ldots, z_{N}\right\} \subset I_{Z}$ with $N \gg 1$ be a set of dense samples, from which an accurate approximation of the expectation $\widetilde{\mathbb{E}}$ (2.4) can be obtained. Let

$$
\gamma_{n}=\left\{z_{i_{1}}, \ldots, z_{i_{n}}\right\} \subset \Gamma_{N}
$$

be a subset of samples. The general procedure of our bi-fidelity algorithm is as follows.

(1) Conduct the low-fidelity sampling at the dense sample set $\Gamma_{N}$ and obtain the low-fidelity approximation $\mu^{L}(\cdot)$ via (2.9).

(2) Select a subset of samples $\gamma_{n} \subset \Gamma_{N}$, where $n \ll N$.

(3) Conduct the high-fidelity computations at the subset $\gamma_{n}$ and obtain the highfidelity solution samples, $u^{H}\left(\gamma_{n}\right)=\left\{u^{H}\left(\cdot, z_{i_{1}}\right), \ldots, u^{H}\left(\cdots, z_{i_{n}}\right)\right\}$.

(4) Construct a bi-fidelity approximation $\mu^{B}=\mu^{B}\left(\mu^{L}, u^{H}\left(\gamma_{n}\right)\right)$.

The key issue lies in Step (2), the selection of the subset $\gamma_{n}$, and Step (4), the construction of the bi-fidelity approximation $\mu^{B}$. We now present the detail of these two steps.

3.1.1. Selection of the subset $\gamma_{n}$. For any set of samples $\delta_{k}=\left\{z_{1}, \ldots, z_{k}\right\}$, we denote

$$
u^{L}\left(\delta_{k}\right)=\left\{u^{L}\left(z_{1}\right), \ldots, u^{L}\left(z_{k}\right)\right\},
$$

and define the corresponding space

$$
U^{L}\left(\delta_{k}\right)=\operatorname{span}\left(u^{L}\left(\delta_{k}\right)\right)=\operatorname{span}\left\{u^{L}\left(z_{1}\right), \cdots, u^{L}\left(z_{k}\right)\right\} .
$$

Similarly, for the high-fidelity model, we define

$$
u^{H}\left(\delta_{k}\right)=\left\{u^{H}\left(z_{1}\right), \ldots, u^{H}\left(z_{k}\right)\right\}, \quad U^{H}\left(\delta_{k}\right)=\operatorname{span}\left(u^{H}\left(\delta_{k}\right)\right) .
$$

Our choice of the $n$-point subset $\gamma_{n}$ follows the procedure proposed in [18, 26]. It is a greedy algorithm that seeks to find the next sample whose corresponding lowfidelity solution is furthest to the space spanned by the existing low-fidelity solution 
set. Starting from a trivial initial choice $\gamma_{0}=\{\}$, we let $\gamma_{k}=\left\{z_{i_{1}}, \ldots, z_{i_{k}}\right\} \subset \Gamma_{N}$ be the $k$-point existing subset in $\Gamma_{N}$. We then find the $(k+1)$-th point by

$$
z_{i_{k+1}}=\underset{z \in \Gamma_{N}}{\operatorname{argmax}} \operatorname{dist}\left(u^{L}(z), U^{L}\left(\gamma_{k}\right)\right), \quad \gamma_{k+1}=\gamma_{k} \cup z_{i_{k+1}},
$$

where the distance function $\operatorname{dist}(g, G)$ between the function $g \in u^{L}\left(\Gamma_{N}\right)$ and the space $G \subset U^{L}\left(\Gamma_{N}\right)$ follows the standard definition. This greedy algorithm can be readily implemented via simple operations of numerical linear algebra.

- Let $\mathbf{W}$ be the Gramian matrix of the low-fidelity solution $u^{L}\left(\Gamma_{N}\right)$, i.e.,

$$
\mathbf{W}=\left(w_{i j}\right)_{1 \leq i, j \leq N}, \quad w_{i j}=\left\langle u^{L}\left(z_{i}\right), u^{L}\left(z_{j}\right)\right\rangle^{L} .
$$

- Apply the pivoted Cholesky decomposition to the matrix $\mathbf{W}$,

$$
\mathbf{W}=\mathbf{P}^{T} \mathbf{L} \mathbf{L}^{T} \mathbf{P}
$$

where $\mathbf{L}$ is lower-triangular and $\mathbf{P}$ is a permutation matrix due to pivoting. This will produce an ordered permutation vector $P=\left(i_{1}, \ldots, i_{N}\right)$, from which we choose the first $n$ points to define $\gamma_{n}=\left\{z_{i_{1}}, \ldots, z_{i_{n}}\right\}$.

More details and properties of the algorithm can be found in [18, 26].

3.1.2. Bi-fidelity approximation. Once the steps (1)-(3) are finished in the main algorithm in Section 3.1, we possess the following

- Low-fidelity solutions at the dense sample set $\Gamma_{N}, u^{L}\left(\Gamma_{N}\right)$, and the corresponding low-fidelity average, $\mu^{L}(2.9)$.

- The subset samples $\gamma_{n} \subset \Gamma_{N}$.

- High-fidelity solutions in the subset $\gamma_{n}, u^{H}\left(\gamma_{n}\right)$.

To construct the bi-fidelity approximation of the average, we first construct the best approximation of $\mu^{L}$ in the low-fidelity space $U^{L}\left(\gamma_{n}\right)$, and then apply the same construction to the high-fidelity space $U^{H}\left(\gamma_{n}\right)$. To accomplish this, we first construct the orthogonal projection operator that mpas the low-fidelity solution space $U^{L}\left(\gamma_{n}\right)$ onto the mean $\mu^{L}$. That is, we define

$$
\mathbb{P}\left[U^{L}\left(\gamma_{n}\right) ; \mu^{L}\right]:=\sum_{k=1}^{n} c_{k} u^{L}\left(\cdot, z_{i_{k}}\right) \approx \mu^{L}(\cdot)
$$

where the coefficients are determined by solving, for each sample $z_{i_{j}} \in \gamma_{n}, j=1, \ldots, n$,

$$
\sum_{k=1}^{n}\left\langle u^{L}\left(z_{i_{k}}\right), u^{L}\left(z_{i_{j}}\right)\right\rangle^{L} c_{k}=\left\langle\mu^{L}, u^{L}\left(z_{i_{j}}\right)\right\rangle^{L}, \quad j=1, \ldots, n,
$$

which is a linear system of equations. The notation $\mathbb{P}\left[U^{L}\left(\gamma_{n}\right) ; \mu^{L}\right]$ implies this operator is constructed by using $\mu^{L}$ and $U^{L}$ constrained on the set $\gamma_{n}$.

Our bi-fidelity approximation of the average is then defined by

$$
\mu^{B}(\cdot):=\mathbb{P}\left[U^{H}\left(\gamma_{n}\right) ; \mu^{L}\right]=\sum_{k=1}^{n} c_{k} u^{H}\left(\cdot, z_{i_{k}}\right),
$$

where the coefficients $c_{k}$ are computed in (3.9). This implies that the bi-fidelity approximation is the output of the same operator $\mathbb{P}$, which is constructed using $\mu^{L}$, with $U^{H}\left(\gamma_{n}\right)$ as input. 
3.2. Error analysis. The algorithm is a straightforward extension of the bifidelity method in $[18,26]$, which seeks to approximate the solution at arbitrary parameter location of $z \in I_{Z}$. (Note that the method in $[18,26]$ does not require probability.) For any $z \in I_{Z}$, we denote

$$
\epsilon(z)=\left\|u^{H}(z)-\mathbb{P}\left[U^{H}\left(\gamma_{n}\right) ; u^{L}(z)\right]\right\|^{H}<\infty
$$

the error bound of the bi-fidelity approximation. The bound was established in Theorem 4.4 of [18]. Its detailed expression is difficult to summarize without invoking too many notations unnecessary for this paper. Interested reader should consult Theorem 4.4 of [18].

Based upon this result, we derive the following error estimate for the bi-fidelity average $\mu^{B}(\cdot)$, compared to the high-fidelity average $\widetilde{\mathbb{E}}\left[u^{H} ; \Gamma_{N}\right]$ (which is not feasible to compute).

THEOREM 3.1. Let $\mu^{B}$ be the bi-fidelity mean (3.10) and $\mu^{H}$ be the high-fidelity mean (2.10). If the assumptions in Theorem 4.4 of [18] are satisfied, then

$$
\left\|\mu^{B}(\cdot)-\mu^{H}(\cdot)\right\|^{H} \leq C_{w}\|\epsilon(z)\|_{\ell^{2}\left(\Gamma_{N}\right)}
$$

where $\epsilon(z)$ is defined in (3.11) and $C_{w}=\|w\|_{\ell_{2}}$ is the 2-norm of the weight vector $w=\left(w_{1}, \ldots, w_{N}\right)$. Furthermore, if all weights are positive, i.e., $w_{i}>0, i=1, \ldots, N$, then

$$
\left\|\mu^{B}(\cdot)-\mu^{H}(\cdot)\right\|^{H} \leq \max _{z \in \Gamma_{N}} \epsilon(z)
$$

Proof. The linearity of the operator $\mathbb{P}$ immediately gives us

$$
\mu^{B}(\cdot)=\mathbb{P}\left[U^{H}\left(\gamma_{n}\right) ; \mu^{L}\right]=\mathbb{P}\left[U^{H}\left(\gamma_{n},\right) ; \widetilde{\mathbb{E}}\left[u^{L} ; \Gamma_{N}\right]\right]=\widetilde{\mathbb{E}}\left[\mathbb{P}\left[U^{H}\left(\gamma_{n}\right) ; u^{L}\right] ; \Gamma_{N}\right]
$$

Then,

$\left\|\mu^{B}-\mu^{H}\right\|^{H}=\left\|\widetilde{\mathbb{E}}\left[\mathbb{P}\left[U^{H}\left(\gamma_{n}\right) ; u^{L}\right]-u^{H} ; \Gamma_{N}\right]\right\|^{H}=\left\|\sum_{i=1}^{N} w_{i}\left(\mathbb{P}\left[U^{H}\left(\gamma_{n}\right) ; u^{L}\left(z_{i}\right)\right]-u^{H}\left(z_{i}\right)\right)\right\|^{H}$.

A straightforward use of the Cauchy-Schwarz inequality completes the proof.

4. Numerical Examples. In this section, we present several numerical examples to illustrate the effectiveness and efficiency of our method. For benchmarking purpose, all examples have relatively small computational cost so that we can run the high-fidelity model many times to compute the reference solution $\mu^{H}$, from which we compute the numerical errors of the bi-fidelity solutions. The errors are reported as the standard $L^{2}$ norm in physical space. Without loss of generality, we employ uniformly distributed random variables in all the examples.

4.1. Function example. We first consider a simple example using a function with known analytical form,

$$
u(x, z)=g\left(x, z+\epsilon z^{2}\right)=\cos \left(x\left(z+\epsilon z^{2}\right)\right), \quad(x, z) \in[-1,1] \times[0,10 \pi] .
$$

To approximate this function we employ polynomials and choose the linear space $V$ to be $\Pi_{K}$, the linear space of polynomial of degrees up to $K$. The orthogonal projection 
of $g$ has the following analytical form,

$$
g_{K}(x, z):=\mathbb{P}_{\Pi_{K}} g=\sum_{k=0}^{K} \hat{g}_{k}(z) \tilde{L}_{k}(x)
$$

where $\tilde{L}_{k}$ is the normalized Legendre polynomial,

$$
\hat{g}_{k}=c_{k} \sqrt{\frac{\pi(2 k+1)}{|z|}} J_{k+1 / 2}(|z|), \quad z \neq 0, \quad c_{k}=\Re\left[e^{(i \operatorname{sgn} z)} i^{k}\right],
$$

and $J_{k}$ is the $k$-th orde Bessel function of the first kind.

We choose the low-fidelity model to be $g_{35}$ and high-fidelity model to be $g_{100}$. The approximation space in physical space $V$ are the piecewise linear polynomials with 500 uniform grids. That is, $V^{L}=V^{H}$. The dense sampling set $\Gamma_{N}=1,000$ Monte Calro points.

In Fig. 4.1 the numerical errors in the first moment (mean) and second moment by the bi-fidelity algorithm are shown, with respect to the number of high-fidelity samples. We clearly observe the very fast error decay with only about 10 samples. For reference the error convergence of the high-fidelity Monte Carlo method (MCS) is also shown, which has the standard $N^{-1 / 2}$ convergence rate. The bi-fidelity method exhibits drastic improvement in performance over the standard MCS. Note that the error of the bi-fidelity method will saturate at about $O(10)$ high-fidelity samples. This is expected as the bi-fidelity method relies on the mapping between $U^{L}\left(\gamma_{n}\right)$ and $\mu^{L}$, as shown in (3.8), whose accuracy is limited by the quality of the low-fidelity model.

4.2. One dimensional stochastic elliptic equation. We now consider an elliptic equation with random diffusivity, a standard benchmark problem in stochastic computing.

$$
\begin{cases}-\left(a(x, z) u_{x}(x, z)\right)_{x}=1, & (x, z) \in(0,1) \times[-1,1]^{d}, \\ u(0, z)=0, & u(1, z)=0,\end{cases}
$$

where the diffusivity field is modeled as

$$
a(x, z)=1+\sigma \sum_{k=1}^{d} \frac{1}{(k \pi)^{2}} \cos (2 \pi k x) z_{k}, \quad d>1 .
$$

Here we let $d=10$ and $\sigma=4$. (The positivity of $a$ is strictly enforced for the choice of the parameters.)

The Chebyshev collocation method is employed to solve the problem in physical space. The low-fidelity model are the solution using 8-point Chebyshev collocation method; whereas the high-fidelity model is based on 128-point Chebyshev collocation method. Consequently, the low-fidelity approximation space $V^{L}$ is different from the high-fidelity approximation space $V^{H}$. Two dense sampling sets are employed to compute the accurate mean. One is the random Monte Carlo sample set with $N=10,000$ points, and the other one is the level 3 Clenshaw-Curtis sparse grids set ([25]). At $d=10$, the sparse grids set has $N=1,581$ points, which have non-uniform and even some negative weights. This is to illustrate the fact that the bi-fidelity method can be applied to any low-fidelity model using the weighted sum form (2.9) for computing the mean. Having these two ways to compute the statistical averages over the dense sets, we thus have two versions of bi-fidelity algorithms. 

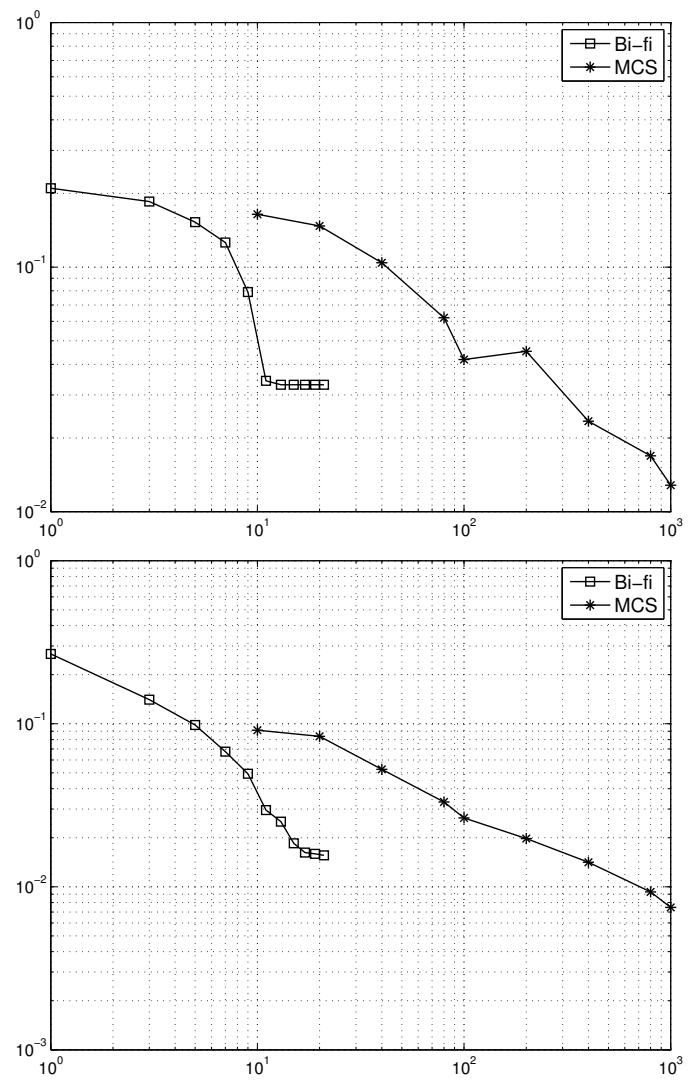

Fig. 4.1. Function example: Decay of errors in the mean (top) and the second moment (bottom) with respect to the number of high-fidelity simulations by the bi-fidelity algorithm and the standard Monte Carlo method.

The convergence of the errors in mean and second moment is shown in Fig. 4.2 with respect to increasing number of high-fidelity simulations, for both the bi-fidelity algorithm based on Monte Carlo simulation and the bi-fidelity method based on sparse grids. For comparison we also show the convergence of the Monte Carlo method and the sparse grids method. Again we observe very fast error decay by the bi-fidelity algorithms. The errors become exceedingly small after only less than 10 high-fidelity samples. This is a drastic improvement over the standard MCS and sparse grids.

4.3. Two dimensional stochastic elliptic equation. We now consider the following 2D stochastic elliptic equation:

$$
\begin{cases}-\nabla \cdot(a(x, y, z) \nabla u)=0, & (x, y) \in(-1,1)^{2}, \\ u(-1, y, z)=-1, \quad u(1, y, z)=1, & u_{y}(x,-1, z)=0, \quad u_{y}(x, 1, z)=0,\end{cases}
$$

where the diffusivity field is modeled via the following Karhunen-Loeve expansion,

$$
a(x, y, z)=1+\sum_{k=1}^{d} \sqrt{\lambda_{k}} \psi_{k}(x, y) z_{k} .
$$

We let $z \in(-1,1)^{d}$ be uniformly distributed random variables and let $\left(\lambda_{k}, \psi_{k}\right)_{k=1}^{d}$ be the eigen-pairs in the Karhunen-Loeve expansion of a random process with the 

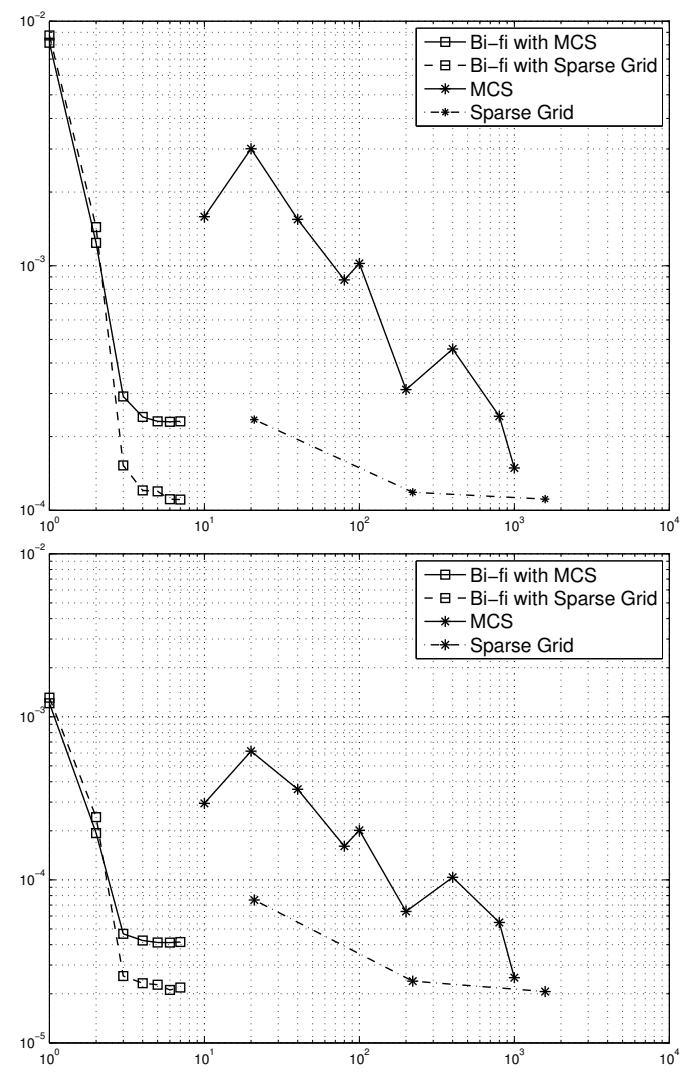

FIG. 4.2. One-dimensional diffusion problem in $d=10$ : Numerical errors in the mean (top) and second moment (bottom) with respect to the number of high-fidelity simulations by the standard Monte Carlo method, the sparse grids method, bi-fidelity method based on Monte Carlo, and bifidelity method based on sparse grids.

following covariance function

$$
C\left(x_{1}, y_{1}, x_{2}, y_{2}\right)=\sigma \exp \left(-\left|x_{1}-x_{2}\right|-\left|y_{1}-y_{2}\right|\right),
$$

with $\sigma=0.3$. The KL expansion has explicitly computable eigenvalue-eigenfunction pairs based on its one-dimensional counterpart (cf., $[13,24])$. We truncate the expansion at $d=17$, which keeps $91.4 \%$ of the total spectral energy.

We construct the following models:

- Model 1. This is a one-dimensional approximation to the two-dimensional problem (4.5). Due to the boundary conditions in (4.5), the problem exhibits certain "near" symmetry. Consequently, we define

$$
\begin{cases}-\left(\hat{a}(x, z) u_{x}(x, z)\right)_{x}=0, & x \in(-1,1) \\ u(-1, z)=-1, & u(1, z)=1\end{cases}
$$

where $\hat{a}(x, z)=a(x, 0, z)=1+\sum_{k=1}^{d} \sqrt{\lambda_{k}} \psi_{k}(x, 0) z_{k}$ is the one-dimensional version (at $y=0$ ) of (4.6). We then employ the $P_{1}$-finite element method with 80 uniform elements to solve (4.7). 
- Model 2. This is the original two-dimensional problem (4.5), where we employ the $P_{1}$ finite elements with a very coarse mesh -128 uniform triangular elements with size $h=1 / 4$.

- High-fidelity model. Here we employ the $P_{1}$ finite element method with a uniform triangular mesh of 12,800 elements with size $h=1 / 40$. This is our high-fidelity model and can well resolve the solution.

We then examine the following bi-fidelity cases.

- Bi-fidelity 1. Here we let Model 1 be the low-fidelity model and construct bi-fidelity approximation in conjunction with the high-fidelity model.

- Bi-fidelity 2. Here we let Model 2 be the low-fidelity model and conduct the bi-fidelity approximation with the high-fidelity model.

In both cases, the dense point set $\Gamma_{N}$ has $N=1,000$ i.i.d samples.

The numerical errors of mean and second moment of "bi-fidelity 1" and "bifidelity 2" approximations are plotted in Figure 4.3. We first notice the fast error decay of the "bi-fidelity 2" case, whose errors decay nearly exponentially fast and reach very low level with only a few high-fidelity simulation runs. This indicates that Model 2, despite its coarse mesh $(h=1 / 4)$, is able to capture the solution variation in the random space reasonably well. On the contrary, the errors in "bi-fidelity 1" saturate at a higher level after a few high-fidelity runs. This is not surprising, as the one dimensional low-fidelity model used in the Bi-fidelity 1 algorithm is rather oversimplified and has lower accuracy. Again, we observe both bi-fidelity approximations show better convergence behavior than the standard Monte Carlo approach.

4.4. Acoustic Horn problem. We now present results for the two-dimensional Helmholtz equation. It is an acoustic horn problem from [26]. The acoustic field is described by the following time-harmonic Helmholtz equation,

$$
\begin{cases}\triangle u+4 u=0, & \\ (2 i+1 / 25) u+\frac{\partial u}{\partial n}=0, & \partial D_{\text {out }} \\ 2 i u+\frac{\partial u}{\partial n}=4 i, & \partial D_{\text {in }} \\ \frac{\partial u}{\partial n}=0, & \partial D_{j}, j=3,8 \\ i \kappa_{j} u+\frac{\partial u}{\partial n}=0, & \text { on other boundaries }\end{cases}
$$

where $i^{2}=-1$ and $\mu=\left(\mu_{1}, \mu_{2}, \mu_{4}, \mu_{5}, \mu_{6}, \mu_{7}, \mu_{9}, \mu_{10}\right) \in[0,1]^{8}$ is modeled as a uniformly distributed random parameter governing the material properties. The geometry is shown in Figure 4.4, where $R=12.5, a=0.5, c=0.1, d=5, l=5$. The boundary condition at $\partial D_{\text {out }}$ is the lowest order Enquist-Majda absorbing boundary condition [10], to reduce the reflection effect due to the artificial boundary. The boundaries $\partial D_{3,8}$ consist of sound-hard materials and the rest of $\partial D_{i}$ mimic sound-soft materials determined by the parameters $\kappa_{i}$.

We solve this problem using $P_{2}$ bubble finite elements using the finite element package Freefem ++ [15]. The low-fidelity model uses a mesh of 2,061 elements; and the high-fidelity model uses a fine mesh of 22,810 elements. The dense point set $\Gamma_{N}$ has $N=5,000$ i.i.d. random points.

The convergence results are shown in Figure 4.5. Again, we observe very fast error convergence in the bi-fidelity simulations. With less than $n=10$ high-fidelity simulations, the errors reduce to the level of the spatial discretization errors. The improvement over the standard Monte Carlo method is obvious.

5. Summary. In this paper we present a bi-fidelity algorithm for approximating the statistical moments of stochastic problems. The method extends the earlier work 

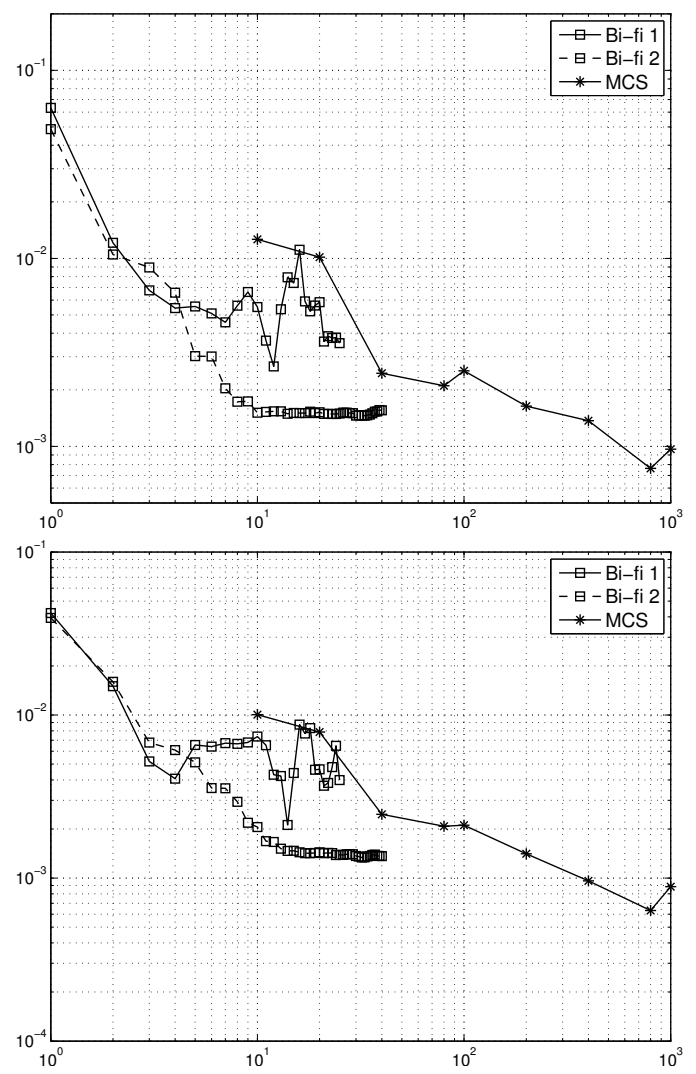

Fig. 4.3. Two dimensional diffusion problem (4.7): Numerical errors of the mean (top) and second moment (bottom) with respect to the number of high-fidelity simulations by the standard Monte Carlo method, the "bi-fidelity 1" and "bi-fidelity 2" method.

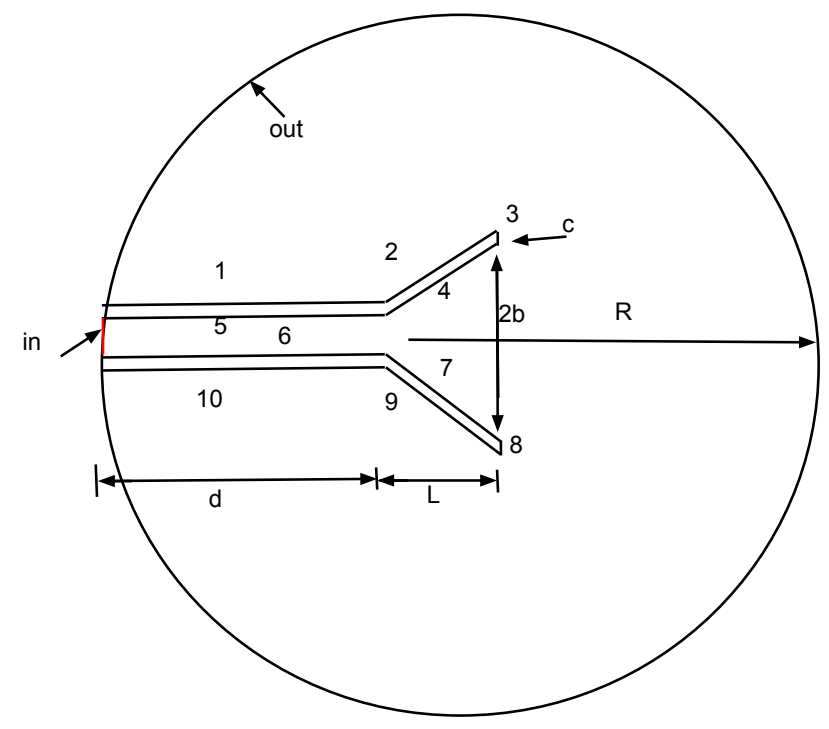

FIG. 4.4. The domain of the acoustic horn problem. 

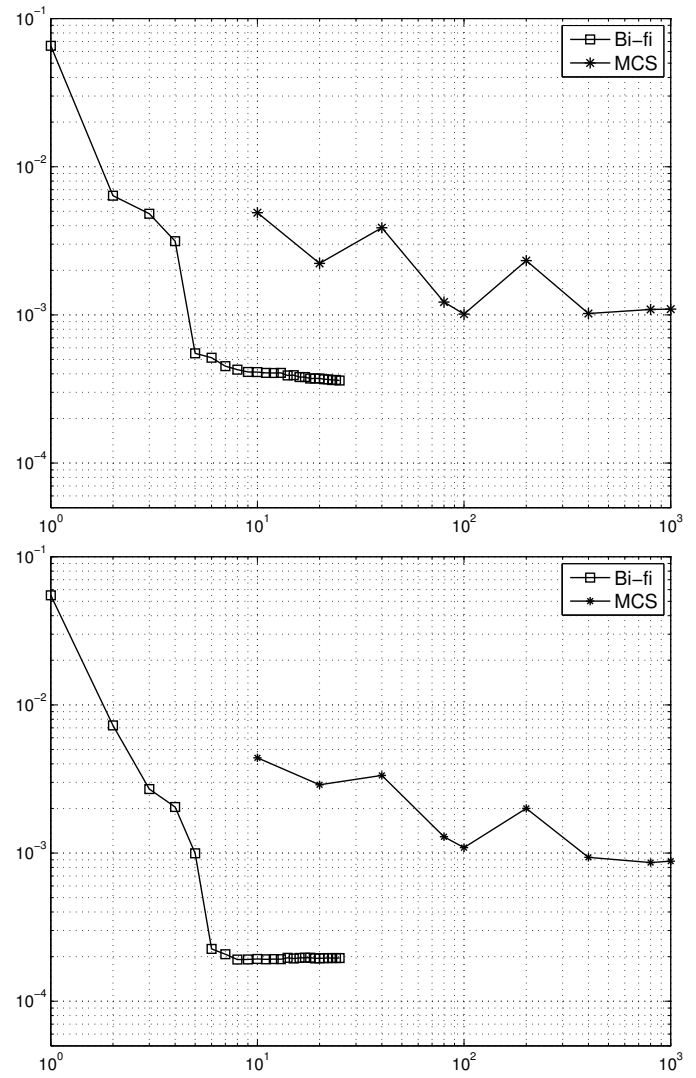

Fig. 4.5. Acoustic horn problem: Numerical errors in mean (top) and second moment (bottom) with respect to the number of high-fidelity simulations by standard Monte Carlo method and bi-fidelity method).

by $[18,26]$, which approximates solutions at given sample locations. The current bi-fidelity method takes advantage of the low cost of the low-fidelity model and the high accuracy of the high-fidelity model. It uses the low-fidelity average and a limited number of high-fidelity simulations to construct a highly accurate approximation of the high-fidelity average. Error bound is established to ensure the method is well behaved. Numerous examples are presented to demonstrate the effectiveness of the bi-fidelity method. In most cases, the current bi-fidelity method is able to produce highly accurate results with only $O(10)$ high-fidelity samples.

\section{REFERENCES}

[1] N. Agarwal and N. Aluru. A domain adaptive stochastic collocation approach for analysis of mems under uncertainties. J. Comput. Phys., 228(20):7662-7688, 2009.

[2] I. Babuska, F. Nobile, and R. Tempone. A stochastic collocation method for elliptic partial differential equations with random input data. SIAM J. Numer. Anal., 45(3):1005-1034, 2007.

[3] A. Barth, A. Lang, and C. Schwab. Multilevel Monte Carlo method for parabolic stochastic partial differential equations. BIT Numer. Math., 53(1):3-27, 2012.

[4] A. Barth, C. Schwab, and N. Zollinger. Multi-level Monte Carlo finite element method for elliptic PDEs with stochastic coefficients. Numer. Math., 119(1):123-161, 2011. 
[5] M. Bieri and C. Schwab. Sparse high order FEM for elliptic sPDEs. Comput. Meth. Appl. Math. Engrg., 198:1149-1170, 2009.

[6] P. Chen, A. Quarteroni, and G. Rozza. Reduced order methods for uncertainty quantification problems. 2015.

[7] K. Cliffe, M. Giles, R. Scheichl, and A. L. Teckentrup. Multilevel monte carlo methods and applications to elliptic pdes with random coefficients. Computing and Visualization in Science, 14(1):3-15, 2011.

[8] A. Doostan and H. Owhadi. A non-adapted sparse approximation of PDEs with stochastic inputs. J. Comput. Phys., 230(8):3015-3034, 2011.

[9] M. Eldred. Recent advances in non-intrusive polynomial chaos and stochastic collocation methods for uncertainty analysis and design. In 50th AIAA/ASME/ASCE/AHS/ASC Structures, Structural Dynamics, and Materials Conference, volume AIAA-2009-2249, 2009.

[10] B. Engquist and A. Majda. Absorbing boundary conditions for numerical simulation of waves. Proceedings of the National Academy of Sciences, 74(5):1765-1766, 1977.

[11] J. Foo, X. Wan, and G. Karniadakis. The multi-element probabilistic collocation method (MEPCM): error analysis and applications. J. Comput. Phys., 227(22):9572-9595, 2008.

[12] B. Ganapathysubramanian and N. Zabaras. Sparse grid collocation methods for stochastic natural convection problems. J. Comput. Phys., 225(1):652-685, 2007.

[13] R. Ghanem and P. Spanos. Stochastic Finite Elements: a Spectral Approach. Springer-Verlag, 1991.

[14] M. Giles. Multilevel Monte Carlo path simulation. Oper. Res., 56(3):607-671, 2008.

[15] F. Hecht. New development in Freefem++. J. Numer. Math., 20(3-4), Jan. 2012.

[16] J. Jakeman and S. Roberts. Stochastic Galerkin and collocation methods for quantifying uncertainty in differential equations: a review. In G. Mercer and A. Roberts, editors, Proceedings of the 14th Biennial Computational Techniques and Applications Conference, CTAC-2008, volume 50 of ANZIAM J., pages C815-C830, Feb. 2009.

[17] X. Ma and N. Zabaras. An adaptive hierarchical sparse grid collocation algorithm for the solution of stochastic differential equations. J. Comput. Phys., 228:3084-3113, 2009.

[18] A. Narayan, C. Gittelson, and D. Xiu. A stochastic collocation algorithm with multifidelity models. SIAM J. Sci. Comput., 36(2):A495-A521, 2014.

[19] A. Narayan and D. Xiu. Stochastic collocation methods on unstructured grids in high dimensions via interpolation. SIAM J. Sci. Comput., 34(3):A1729-A1752, 2012.

[20] F. Nobile, R. Tempone, and C. Webster. A sparse grid stochastic collocation method for partial differential equations with random input data. SIAM J. Numer. Anal., 46(5):2309-2345, 2008 .

[21] A. Teckentrup, R. Scheichl, M. Giles, and E. Ullmann. Further analysis of multilevel monte carlo methods for elliptic pdes with random coefficients. Numerische Mathematik, 125(3):569600, 2013.

[22] F. Vidal-Codina, N.-C. Nguyen, M. Giles, and J. Peraire. A model and variance reduction method for computing statistical outputs of stochastic elliptic partial differential equations. arXiv preprint arXiv:1409.1526, 2014.

[23] D. Xiu. Efficient collocational approach for parametric uncertainty analysis. Comm. Comput. Phys., 2(2):293-309, 2007.

[24] D. Xiu. Numerical methods for stochastic computations. Princeton Univeristy Press, Princeton, New Jersey, 2010.

[25] D. Xiu and J. Hesthaven. High-order collocation methods for differential equations with random inputs. SIAM J. Sci. Comput., 27(3):1118-1139, 2005.

[26] X. Zhu, A. Narayan, and D. Xiu. Computational aspects of stochastic collocation with multifidelity models. SIAM/ASA Journal on Uncertainty Quantification, 2(1):444-463, 2014. 